\title{
Effect of robot arm reach training on upper extremity functional movement in chronic stroke survivors: a preliminary study
}

\author{
Ki Hun Cho ${ }^{a}$, Won-Kyung Song ${ }^{b}$ \\ ${ }^{a}$ Department of Physical Therapy, Korea National University of Transportation, Jeungpyeong, Republic of Korea \\ ${ }^{\mathrm{b}}$ Department of Rehabilitative and Assistive Technology, National Rehabilitation Research Institute, National Rehabilitation Center, Seoul, \\ Republic of Korea
}

Objective: The purpose of this study was to investigate the effect of robot arm reach training on upper extremity functional movement in chronic stroke survivors.

Design: One group pretest-posttest design.

Methods: Thirteen chronic stroke survivors participated in this study. Robot arm reach training was performed with a Whole Arm Manipulator (WAM) and a 120-inch projective display to provide visual and auditory feedback. During the robotic arm reach training, WAM provided gravity compensation and assist-as-needed (AAN) force according to the robot control mode. When a participant could not move the arm toward the target for more than 2 seconds, WAM provided AAN force to reach the desired targets. All patients participated in the training for 40 minutes per day, 3 times a week, for 4 weeks. Main outcome measures were the Fugl-Meyer Assessment (FMA), Action Research Arm Test (ARAT) and Box and Block Test (BBT) to assess upper extremity functional movement.

Results: After 4 weeks, significant improvement was observed in upper extremity functional movement (FMA: 42.15 to 46.23, BBT: 12.23 to $14.00, p<0.05)$. In the subscore analysis of the FMA upper extremity motor function domains, significant improvement was observed in upper extremity and coordination/speed units $(p<0.05)$. However, there were no significant differences in the ARAT.

Conclusions: This study showed the positive effects of robot arm reach training on upper extremity functional movement in chronic stroke survivors. In particular, we confirmed that robot arm reach training could have a positive influence by leading to improvement of motor recovery of the proximal upper extremity.

Key Words: Robotics, Stroke, Upper extremity

\section{Introduction}

Stroke can lead to various disabilities depending on the degree and location of brain injury [1]. Moreover, stroke is a main cause of chronic upper extremity movement impairment. Most stroke survivors have functional disabilities in daily living activity performances associated with use of the upper extremity [2]. Only a small portion of individuals experience a complete functional recovery after a stroke, with $55 \%$ to $75 \%$ of survivors left with lingering upper extremity impairments as well as activity of daily living and community participation restrictions at 6 months after onset [3]. Thus, improving upper limb capacity of stroke survivors is one of the most important challenges for clinicians and researchers [4].

Over the years, various therapeutic approaches, such as

Received: 31 May, 2019 Revised: 19 June, 2019 Accepted: 19 June, 2019

Corresponding author: Won-Kyung Song (ORCID https://orcid.org/0000-0002-9884-0467)

Department of Rehabilitative and Assistive Technology, National Rehabilitation Research Institute, National Rehabilitation Center, 58 Samgaksan-ro, Gangbuk-gu, Seoul 10122, Republic of Korea

Tel: 82-2-901-1901 Fax: 82-2-901-1910 E-mail: wonksong@gmail.com

(c) This is an Open-Access article distributed under the terms of the Creative Commons Attribution Non-Commercial License (http://creativecommons.org/licenses/ by-nc/4.0) which permits unrestricted non-commercial use, distribution, and reproduction in any medium, provided the original work is properly cited.

Copyright $\odot 2019$ Korean Academy of Physical Therapy Rehabilitation Science 
task-specific training [5], constraint-induced movement therapy [6], virtual reality training [7], and functional electrical stimulation [8] have been used to improve upper extremity function. Among the potential therapeutic approach used to aid recovery in stroke survivors, the robotic system has recently been developed to improve upper extremity movement [9]. The robotic system enables users to participate in motivational, repetitive and intensive tasks. According to a previous review, robotic systems have the potential to improve arm function and basic activities of daily living [4], and another systematic review reported that the robot-assisted training is effective in improving upper extremity motor functional recovery of persons with stroke [10]. However, although robotic systems have been used consistently for upper limb rehabilitation after stroke [11], scientific evidence of their benefits is still insufficient. In particular, scientific evidence for the various training modes used in upper limb robot rehabilitation is lacking.

Thus, the purpose of the current study was to assess whether the robot arm reach training with assistance force could improve upper extremity functional movement in chronic stroke survivors. In this study, the Fugl-Meyer Assessment (FMA) subscore analysis was particularly performed to investigate the change of motor function after robot arm reach training with assistance force. We hypothesized that stroke survivors would show enhancements in upper extremity functional movement after 4 weeks of robot arm reach training with assistance force. The goal of present study was to provide a reference point for a future randomized controlled trial.

\section{Methods}

\section{Subjects}

Sixteen chronic stroke survivors participated in this study. Participants were recruited by the following inclusion and exclusion criteria. The inclusion criteria were as follows: 1) hemiparesis from a single stroke occurring at least six months prior, 2) Mini-Mental State Examination scores of 24 or higher, 3) no known musculoskeletal conditions that would have affect the ability to sit safely, and 4) absence of hemispatial neglect. The exclusion criteria were as follows: 1) participation with rehabilitation programs, 2) participation with shoulder subluxation or pain, or 3) spasticity (Modified Ashworth Scale score>2). Participants were recruited from the local community who were not participating in any rehabilitation programs. After screening, 3 pa- tients were excluded in this study because they did not meet the inclusion criteria.

\section{Procedures}

This was a single-group pre- and post-test design study with an aim of investigating the effect of robot arm reach training on upper extremity functional movement in chronic stroke survivors. The objectives and experimental procedures of study were explained to all the participants, and they had voluntarily signed the informed consent forms. Ethical approval for the study was granted by the Korea National Rehabilitation Center Institutional Review Board (NRC-2017-01-011).

All participants participated in the training three times per week for 4 weeks. The duration of a single training session was 40 minutes with the same assistants. All participants completed the training sessions, and were assessed for functional movement using the FMA, Action Research Arm Test (ARAT) and Box and Block Test (BBT). The post-test was performed three days after the end of the intervention. In addition, all measurements were performed by an occupational therapist and a physical therapist.

\section{Experimental protocol: robot arm training with an assis- tance force}

The robot arm reach training with an assistance force was performed on a test bed. The setting of the test bed was duplicated as reported in previous studies [12-14]. The robot arm reach training with an assistance force consisted of a Whole Arm Manipulator (WAM) (Barrett Technology, Inc., Newton, MA, USA) with seven degrees of freedom and one a 120-inch projective display.

A projective display in front of the test bed provided suitable visual and auditory feedback to the subjects. Participants performed the robot arm reach training with the WAM in sitting position with a trunk-fixation belt. The trunk-fixation belt was used to minimize compensatory movement (Figure 1).

During the robot arm reach training, red and gray balls appeared on the display, and the subjects performed reaching movements toward the targets in six directions (targets 1-6, Figure 2) [13]. The red ball was linked to the user's upper limb movements. When the red and gray balls matched, auditory feedback (ding-dong sound) was provided to user.

During the robot arm reach training, the WAM provided additional assistance force. Additional assistance force consisted of gravity compensation and assist-as-needed (AAN) 


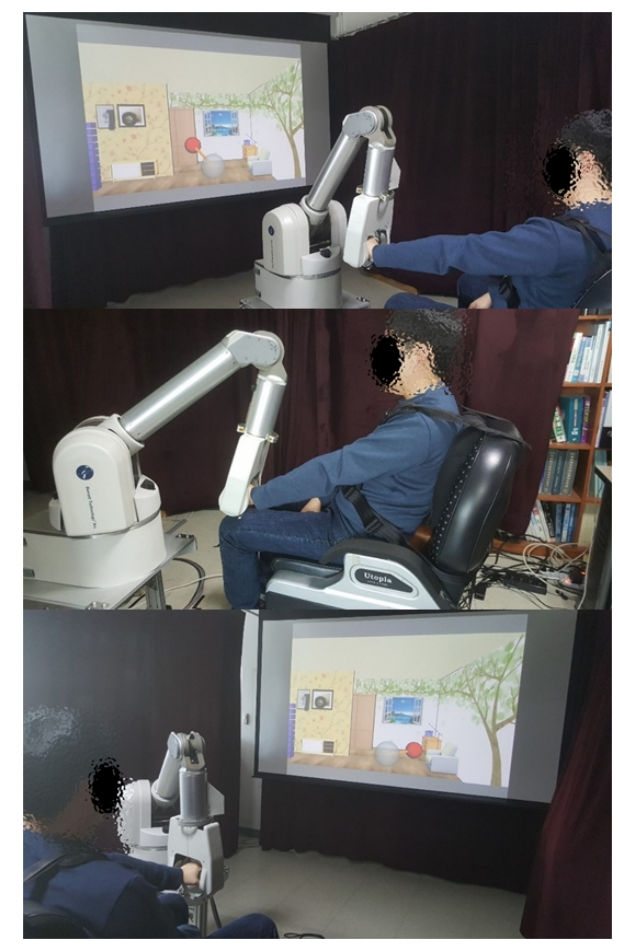

Figure 1. Setting for the robotic arm training with an assistance force program. The training test bed consisted of a Whole Arm Manipulator (WAM) and a projective display. The WAM provides a highly back-drivable motion that helps the user reach the desired sphere using point-to-point movements. A 120 -inch projective display attached to the front of the test bed was used to provide suitable visual and auditory feedback to the user.

force [14]. Gravity compensation was provided continuously during robot arm reach training, and an AAN force was selectively provided depending on user's reaching performance. When the participant could not move the arm toward the target for more than 2 seconds, the WAM provided AAN force to reach the desired targets (Figure 2) [13]. The AAN force was introduced temporarily to assist the lack of reaching movement. Additional AAN was restricted to 4 Newtons for safety according the recommendation of clinical experts. While performing the robot arm reach training with an assistance force, an assistant stood nearby and emergency stop devices were installed to prevent accidents due to fatigue. All patients participated in the robotic arm training with an assistance force program for 40 minutes per day, 3 times per week, for 4 weeks.

\section{Outcome measures}

The FMA, ARAT and the BBT was used to measure functional movement of the upper extremity.

The FMA is an index to assess the sensorimotor impair-

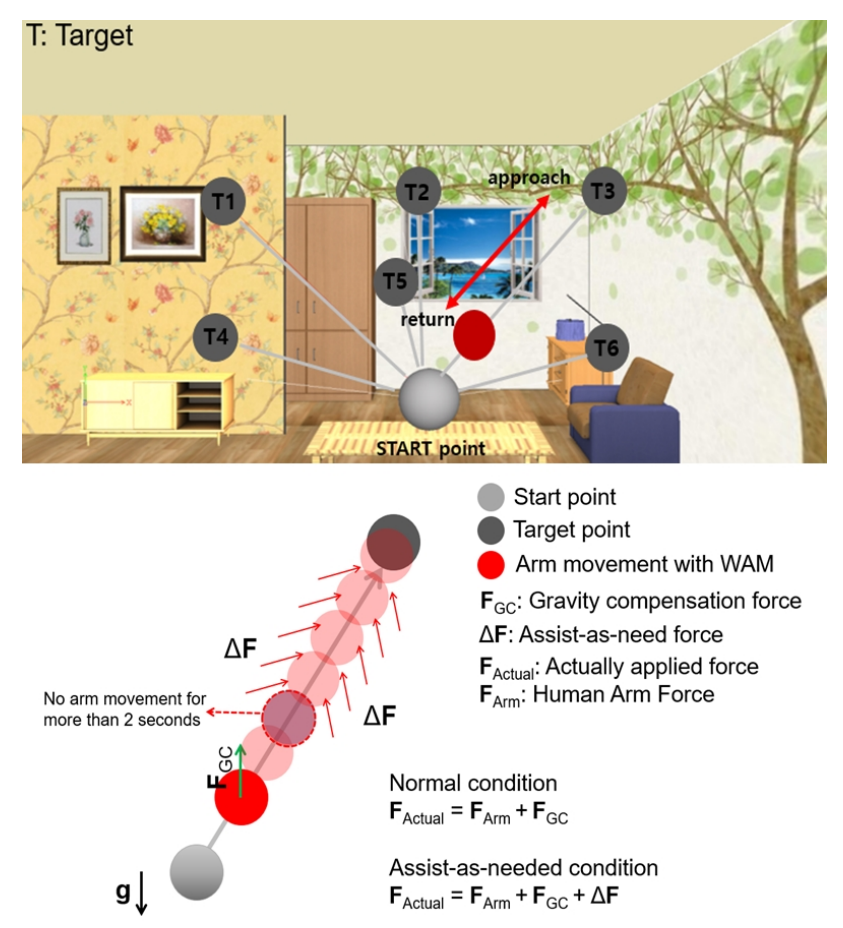

Figure 2. Additional assistance force (gravity compensation and AAN force) control strategy: when the subject performed robotic arm training, gravity compensation was provided continuously, and an AAN force was only applied when the subject could not move the arm for more than 2 seconds. AAN: assist-as-needed, WAM: Whole Arm Manipulator. Data from the article of Cho and Song (J Phys Ther Sci 2016;28:495-9) [13].

ment in individuals who have had stroke. In this study, only motor function of the upper extremity domain (maximum score $=66$ ) was assessed [15].

The ARAT is designed to evaluate upper limb functional disability through the assessment of four basic movements (primary grasp, grip, pinch, and gross movements). Each test is graded on a four-point scale, from 0 to 3 , yielding a maximum score of 57 [16].

The BBT evaluates gross manual dexterity in a wide range of populations, including stroke survivors. The BBT is a quick and simple test. During the tests, the subject was instructed to move as many blocks as possible from one side to the other within 1 minute using only the tested hand [17].

\section{Data analysis}

Data analysis was performed using the IBM SPSS Statistics ver. 21.0 software (IBM Co., Armonk, NY, USA). The Shapiro-Wilk test was used to confirm the normal distribution of all outcome variables. Since all variables were normally distributed the paired t-test was used to determine between which comparisons the differences existed. All out- 
comes are expressed as mean values and standard deviations. Statistical significance was accepted for $p<0.05$.

\section{Results}

A summary of the general characteristics of the 13 subjects is shown in Table 1. Table 2 and Figure 2 show the changes in upper extremity functional movement according to the robot arm reach training with an assistance force. After 4 weeks of the robot arm reach training with an assis-

Table 1. General characteristic of the subjects

$(\mathrm{N}=13)$

\begin{tabular}{lc}
\hline \multicolumn{1}{c}{ Parameter } & Value \\
\hline Sex & \\
Male & $10(76.9)$ \\
Female & $3(23.1)$ \\
Etiology & \\
Infarction & $5(38.5)$ \\
Hemorrhage & $8(61.5)$ \\
Paretic side & \\
Right & $9(69.2)$ \\
Left & $4(30.8)$ \\
MAS-UE (1/1+/2) & $6 / 6 / 1$ \\
Brunstrom stage_UE (2/3/4/5/6) & $1 / 1 / 3 / 5 / 3$ \\
MRC-EE (3/4/5) & $1 / 4 / 8$ \\
MRC-SHF (3/4/5) & $2 / 3 / 8$ \\
Age (y) & $59.84(6.22)$ \\
Time since stroke (y) & $12.69(9.26)$ \\
Height (cm) & $166.61(10.98)$ \\
weight (kg) & $68.95(6.42)$ \\
MMSE (scores) & $27.00(2.58)$ \\
\hline
\end{tabular}

Values are presented as number (\%), number only, or mean (SD). MAS-UE: Modified Ashworth Scale for Upper Extremity, MRC-EE: Medical Research Council Scale for muscle strength on Elbow Extension, MRC-SHF: Medical Research Council Scale for muscle strength on Shoulder Flexion, MMSE: Mini-Mental State Examination.

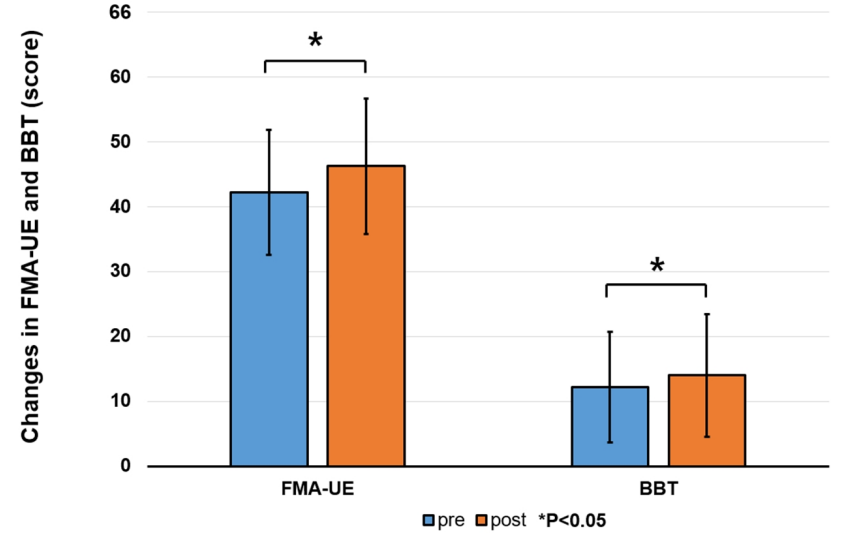

Figure 3. Changes in functional ability of the upper extremity. After 4 weeks of robotic arm training with an assistance force, significant improvement was observed in FMA-UE and BBT. FMA-UE: Fugl-Meyer Assessment of Upper Extremity motor function domain, BBT: Box and Block Test.

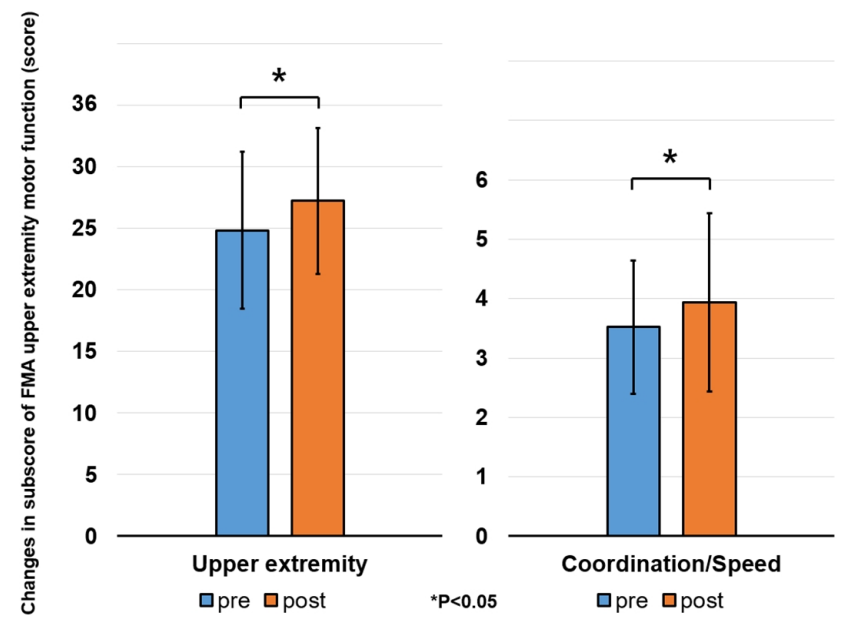

Figure 4. Changes in subscores of FMA upper extremity motor function. After 4 weeks of robotic arm training with an assistance force, significant improvement was observed in upper extremity and coordination/speed. FMA: Fugl-Meyer Assessment.

Table 2. Changes in upper extremity functional movement

$(\mathrm{N}=13)$

\begin{tabular}{lcccr}
\hline \multicolumn{1}{c}{ Parameters } & Pre-test & Post-test & Changes & $\mathrm{t}(p)$ \\
\hline FMA-UE total (score) & $42.15(9.63)$ & $46.23(10.47)$ & $4.07(4.90)$ & $-2.966(0.011)$ \\
Upper extremity (score) & $24.84(6.36)$ & $27.23(5.94)$ & $2.38(3.27)$ & $-2.622(0.022)$ \\
Wrist & $5.53(2.18)$ & $5.53(2.14)$ & $0.00(0.40)$ & $0.000(1.000)$ \\
Hand & $8.69(4.88)$ & $9.76(5.11)$ & $1.07(2.75)$ & $-1.411(0.184)$ \\
Coordination/speed & $3.30(0.63)$ & $3.92(1.25)$ & $0.61(0.96)$ & $-2.309(0.040)$ \\
ARAT (score) & $32.15(20.61)$ & $34.38(20.94)$ & $2.23(3.78)$ & $-2.123(0.055)$ \\
BBT (score) & $12.23(8.51)$ & $14.00(9.49)$ & $1.76(1.92)$ & $-3.320(0.006)$ \\
\hline
\end{tabular}

Values are presented as mean (SD).

FMA-UE total: Fugl-Meyer Assessment of Upper Extremity motor function domains, ARAT: Action Research Arm Test, BBT: Box and Block Test. 
tance force, significant improvement was observed in the FMA and BBT (FMA: 42.15 to 46.23, BBT: 12.23 to 14.00 , $p<0.05$, Figure 3 ). In particular, significant improvement was observed in upper extremity and coordination/speed units as seen in the subscore analysis of the FMA for upper extremity motor function domains (upper extremity unit: 24.84 to 27.23 , coordination/speed unit: 3.30 to 3.92 , $p<0.05$, Table 2 and Figure 4). However, there were no significant differences in the ARAT.

\section{Discussion}

Stroke is a main cause of movement dysfunction and upper extremity functional impairments are particularly present in $>50 \%$ of stroke survivors [3]. In addition, stroke is a risk factor associated with chronic impairment of upper limb function, and improvement of upper limb ability is known to provide opportunities to participate in the community [12, 13]. Thus, restoration of upper limb function is an essential goal of stroke rehabilitation.

The present study was performed to investigate the effect of the robot arm reach training with an assistance force on upper extremity functional movement in stroke survivors. After four weeks of robot arm reach training with an assistance force, significant improvements in upper extremity functional movement (FMA-UE and BBT) were observed in stroke survivors. In stroke rehabilitation, providing appropriate assistance when needed is important to induce active participation [18]. In this study, when a subject could not move the arm toward the target for 2 seconds, they were provided with AAN force to reach the desired targets. Through this additional assistance force, subjects were able to maintain repeated reaching movements from the home position to the target position. Thus, it is considered that an AAN force, provided only when needed based on the user's reach performance, induced enhancements in active participation and functional re-education. In addition, it is considered that intensive repetitive reaching movements may have led to the improvement of agility of the upper extremity.

As another interesting result, a significant improvement was observed in upper extremity and coordination/speed units among the FMA upper extremity motor recovery domains. An individual analysis for distal and proximal parts of upper limb during reaching movements is needed to investigate the training effect [19]. Generally, rehabilitation robots are classified into end-effector and exoskeleton types. In particular, an end-effector robot interacts with the user through the single distal attachment of the hand without being attached to the upper limb. Thus, it is possible to provide free motion to the user's upper extremity in the three-dimensional space, thereby inducing coordinative movements. The WAM used in this study is an end-effector type robot and it was set at seven degrees of freedom for the shoulder, elbow, and wrist joints. Thus, it is considered that the free motion of the upper limb provided by the WAM has led to the improvement of upper extremity and coordination/speed items. In this study, no significant improvement was observed in hand and wrist items. The participants included in this study were chronic stroke survivors (onset duration: 12 years). Most of the participants had the WAM fixated onto their hand using a strap during the robot arm reach training because they were unable to perform a hand grasp at the beginning of the study. For this reason, it is considered that the robot arm reach training could not induce notable improvements in hand and wrist functions.

This study has several limitations. First, since the aim of this study was to assess whether robot arm reach training with an assistance force could improve upper extremity function in chronic stroke survivors, there was no separate control group. Thus, randomized controlled trials should be performed in the future. Second, this study included only high-functioning persons with stroke. Thus, the results of the current study cannot be generalized to all stroke survivors.

In conclusion, this study showed the positive effects of robot arm reach training with an assistance force on upper extremity functional movement in hemiparetic stroke survivors. In particular, it was confirmed that robot arm reach training with AAN force could positively influence the improvement of motor recovery of the proximal upper extremity proximal.

\section{Acknowledgements}

This study was supported by the Research Program (NRCTR-IN17005, NRCTR-IN18006, NRCTR-IN19006) of the Korea National Rehabilitation Center, Ministry of Health and Welfare, Republic of Korea. In addition, we are grateful to Dr. Joon-Ho Shin and Mr. Jun-Yong Song from the National Rehabilitation Center, Korea.

\section{Conflict of Interest}

The authors declared no potential conflicts of interest 
with respect to the authorship and/or publication of this article.

\section{References}

1. Wade DT, Hewer RL. Functional abilities after stroke: measurement, natural history and prognosis. J Neurol Neurosurg Psychiatry 1987;50:177-82.

2. Jørgensen HS, Nakayama H, Raaschou HO, Olsen TS. Stroke. Neurologic and functional recovery the Copenhagen Stroke Study. Phys Med Rehabil Clin N Am 1999;10:887-906.

3. Kwakkel G, Kollen B, Lindeman E. Understanding the pattern of functional recovery after stroke: facts and theories. Restor Neurol Neurosci 2004;22:281-99.

4. Pollock A, Farmer SE, Brady MC, Langhorne P, Mead GE, Mehrholz J, et al. Interventions for improving upper limb function after stroke. Cochrane Database Syst Rev 2014;(11): CD010820.

5. Bayona NA, Bitensky J, Salter K, Teasell R. The role of task-specific training in rehabilitation therapies. Top Stroke Rehabil 2005;12:58-65.

6. Wu CY, Chen YA, Lin KC, Chao CP, Chen YT. Constraint-induced therapy with trunk restraint for improving functional outcomes and trunk-arm control after stroke: a randomized controlled trial. Phys Ther 2012;92:483-92.

7. Kowalczewski J, Chong SL, Galea M, Prochazka A. In-home tele-rehabilitation improves tetraplegic hand function. Neurorehabil Neural Repair 2011;25:412-22.

8. Alon G, Levitt AF, McCarthy PA. Functional electrical stimulation (FES) may modify the poor prognosis of stroke survivors with severe motor loss of the upper extremity: a preliminary study. Am J Phys Med Rehabil 2008;87:627-36.

9. Veerbeek JM, Langbroek-Amersfoort AC, van Wegen EE, Meskers CG, Kwakkel G. Effects of robot-assisted therapy for the upper limb after stroke: a systematic review and meta-analysis. Neurorehabil Neural Repair 2017;31:107-21.
10. Bertani R, Melegari C, De Cola MC, Bramanti A, Bramanti P, Calabrò RS. Effects of robot-assisted upper limb rehabilitation in stroke patients: a systematic review with meta-analysis. Neurol Sci 2017;38:1561-9.

11. Mehrholz J, Platz T, Kugler J, Pohl M. Electromechanical and robot-assisted arm training for improving arm function and activities of daily living after stroke. Stroke 2009;40:e392-3.

12. Cho KH, Song WK. Robot-assisted reach training for improving upper extremity function of chronic stroke. Tohoku J Exp Med 2015;237:149-55.

13. Cho KH, Song WK. Feedback training using a non-motorized device for long-term upper extremity impairment after stroke: a single group study. J Phys Ther Sci 2016;28:495-9.

14. Cho KH, Song WK. Robot-assisted reach training with an active assistant protocol for long-term upper extremity impairment poststroke: a randomized controlled trial. Arch Phys Med Rehabil 2019;100:213-9.

15. Sullivan KJ, Tilson JK, Cen SY, Rose DK, Hershberg J, Correa A, et al. Fugl-Meyer assessment of sensorimotor function after stroke: standardized training procedure for clinical practice and clinical trials. Stroke 2011;42:427-32.

16. Yozbatiran N, Der-Yeghiaian L, Cramer SC. A standardized approach to performing the action research arm test. Neurorehabil Neural Repair 2008;22:78-90.

17. Desrosiers J, Bravo G, Hébert R, Dutil E, Mercier L. Validation of the Box and Block Test as a measure of dexterity of elderly people: reliability, validity, and norms studies. Arch Phys Med Rehabil 1994;75:751-5.

18. Frisoli A, Procopio C, Chisari C, Creatini I, Bonfiglio L, Bergamasco $\mathrm{M}$, et al. Positive effects of robotic exoskeleton training of upper limb reaching movements after stroke. J Neuroeng Rehabil 2012;9:36.

19. Hsieh YW, Lin KC, Wu CY, Shih TY, Li MW, Chen CL. Comparison of proximal versus distal upper-limb robotic rehabilitation on motor performance after stroke: a cluster controlled trial. Sci Rep 2018;8:2091. 